\title{
The possible explanation of low-frequency noise of pulsars in globular clusters
}

\author{
Tatiana I. Larchenkova ${ }^{1}$ and Sergei M. Kopeikin ${ }^{2}$ \\ ${ }^{1}$ Astro Space Center of P.N.Lebedev Physical Institute, Leninskii Prospect 53, Moscow \\ 117924, Russia \\ email: tanya@lukash.asc.rssi.ru \\ ${ }^{2}$ Department of Physics and Astronomy, University of Missouri-Columbia, \\ Columbia, MO 65211, USA \\ email: kopeikins@missouri.edu
}

\begin{abstract}
The low-frequency (LF) timing noise contaminates residuals of time-of-arrivals (TOAs) of pulsar signals on long time intervals and in some cases can be explained by external astronomical factors not intrinsically related to the pulsar itself. A number of millisecond pulsars located in globular clusters show the LF noise presence in their rotational phase. We discuss a possible origin of this noise as caused by random time variations in the Shapiro time delay caused by flybys of stars of the globular cluster passing near the pulsar line of sight. The Shapiro time delay is integrated over space of parameters characterizing statistical ensemble of stars in the globular cluster and its long-term indeterministic time variation is obtained. We use this result for numerical simulations of the autocovariance function of the LF timing noise and show it can be used for the measuring of the density profile distribution in the globular cluster.
\end{abstract}

Keywords. Pulsars, globular clusters, relativity

The long-term monitoring of millisecond pulsars has revealed the presence of non-white component of the LF noise of an astrophysical origin being not intrinsically related to the pulsar itself. One of the problems of current pulsar timing analysis is the detection of such a correlated ("red") noise in the spectrum of its TOA residuals and the measuring of its amplitude and spectral index. Now a high accuracy of pulsar timing observations and the exceptionally precision of the data processing algorithms allow to solve this problem. The globular cluster pulsars show the LF noise presence in their rotational phase. We propose the Shapiro effect, i.e. the relativistic time delay in the propagation of pulsar signals passing through the gravitational field of the astrophysical body (the stars of the globular cluster), as a possible cause of the LF timing noise of pulsars in globular clusters. For example, knowledge of the theoretical power spectrum of this effect and the comparison with the observational spectrum allow to obtain the additional information about the globular cluster structure and extend the mass function aside the low mass.

The exact expressions for the relativistic time delay of propagation of electromagnetic signals passing through the non-stationary gravitational field have been derived by Kopeikin \& Schafer (1999) in the form of instantaneous function of the retarded time. As appears from this paper the position of gravitating bodies (stars) should not be taken at the moment of the impulse emission but at its corresponding retarded moment of time.

Let us assume that the origin of the coordinate system is at the barycenter of the globular cluster. And $t_{0}$ - moment of emission of photon, $\vec{x}$ - barycentric coordinates of the observer, $\vec{x}_{a}$ - barycentric coordinates of a-th star, $\vec{x}_{0}$ - barycentric coordinates of the pulsar. Then we can obtain the analytic expression of the stochastic noise process as the result of random time variations in the Shapiro time delay caused by flybys of 

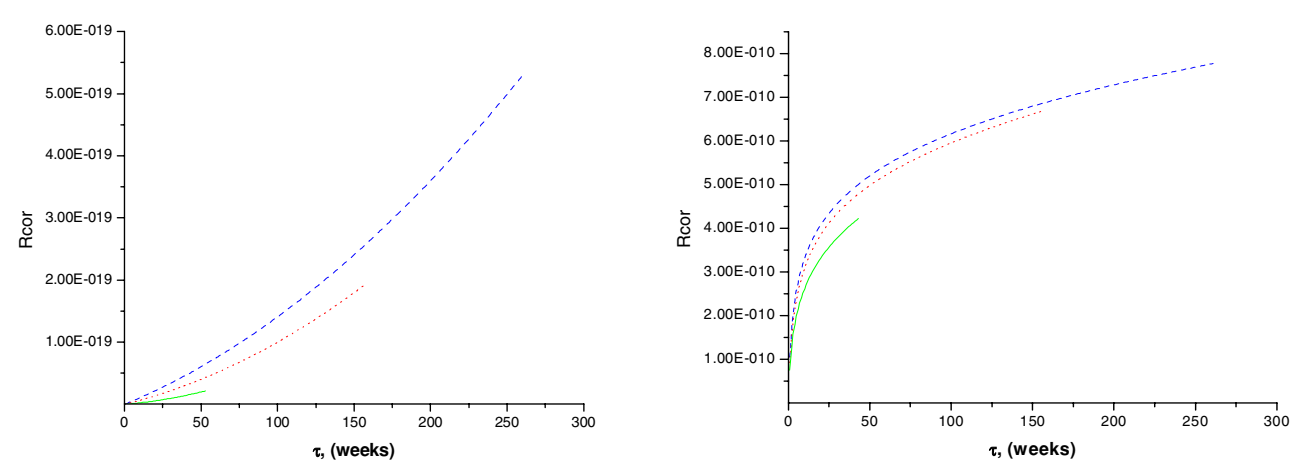

Figure 1. (a) The autocovariance function of stochastic Shapiro effect as a function of the time for the isothermal-sphere model. (b) The same as in the panel (a), but in the case of small impact parameters $\left(\left|\vec{d}_{a}\right|<1 A U\right)$. The pulsar is located in the cluster center for both cases

stars of the globular cluster passing near the pulsar line of sight (see Larchenkova \& Kopeikin (2005) for details). Neglecting all small aberration terms $(v / c)$ we have derived the formula for the stochastic relativistic time delay of the emission of pulsars located in the globular cluster

$\varepsilon(t)=\sum_{a=1}^{N} \frac{2 G M_{a}}{c^{3}} \cdot\left[\ln \left(1+2(t-T) \frac{\vec{d}_{a}\left(T_{0}\right) \vec{v}_{a}\left(T_{0}\right)}{\vec{d}_{a}\left(T_{0}\right) \vec{x}_{a}\left(t_{0}\right)}\right)-\ln \left(1+\frac{\left(-\vec{q}-\vec{K}_{0}\right) \vec{V}_{0 a}\left(T_{0}\right)}{Q_{0 a}+\vec{K}_{0} \vec{Q}_{0 a}}(t-T)\right)\right]$

where $G$ is the gravitational constant, $c$ is the light velocity, $T$ is the beginning of observations, $T_{0}$ defines the moment of the impulse emission corresponding to the time $T, M_{a}$ and $\vec{v}_{a}$ are the mass and the velocity of a-th star, $\vec{K}_{0}$ is the unit vector, $\vec{V}_{0 a}=\vec{v}_{0}\left(T_{0}\right)-\vec{v}_{a}\left(T_{0}\right)$, $\vec{Q}_{0 a}=\vec{x}_{a}\left(T_{0}\right)-\vec{x}_{0}\left(T_{0}\right), \vec{q}=\frac{\vec{Q}_{0 a}}{\left|\vec{Q}_{0 a}\right|}, \vec{v}_{0}$ - the pulsar velocity, $\vec{d}_{a}$ - the impact parameter.

Assuming that the globular cluster stars are uncorrelated, it was shown that the stochastic Shapiro effect manifests itself by the LF noise of the pulsar rotational frequency for large values of the impact parameter. For the pulsar located in the globular cluster 47 Tuc the numerical analysis was done in the case of small values of impact parameters (the short-term part of this stochastic process). The isothermal sphere model and King model for the cluster density distribution, the Salpeter function for the mass distribution of the cluster stars and the Maxwell function for the velocity distribution in a star cluster were used in our analysis. The numerical simulations show that the autocovariance function of the stochastic Shapiro effect can be good approximated by a second-power polynomial in the case of large impact parameters (see Figure $1 a$ ). The power spectral index is equal to $\sim-1.8$ for the King model as well as for the isothermalsphere model. The autocovariance function of the stochastic Shapiro effect for small impact parameters has logarithmic behavior and can be interpreted by flicker noise (see Figure $1 b$ ). The power spectral index for this case is $\sim-1.5$.

We have shown the rotational phase modulation of the pulsar located in globular cluster can be explained by relativistic time delay fluctuations of the pulsar signal propagation in the gravitational field of arbitrarily moving globular cluster stars.

\section{References}

Kopeikin, S.M. \& Schafer, G. 1999, Physical Review D 60, 124002

Larchenkova, T.I. \& Kopeikin, S.M. 2005, Astronomy Letters 31, N12 